\title{
MEDIUM FORMAT CAMERA EVALUATION BASED ON THE LATEST PHASE ONE TECHNOLOGY
}

\author{
T.Tölg ${ }^{\text {a, }}$ G. Kemper ${ }^{\text {b }}$ D. Kalinski ${ }^{\mathrm{c}}$ \\ a Phase One / Germany - tto@phaseone.com \\ ${ }^{\mathrm{b}}$ GGS GmbH, Speyer / Germany - kemper@ggs-speyer.de \\ c Phase One / Israel - dov@ phaseone.com
}

Commission I, WG I/2

KEY WORDS: Camera evaluation, calibration, mapping, accuracies, CMOS, CCD, FMC

\begin{abstract}
:
In early 2016, Phase One Industrial launched a new high resolution camera with a 100 MP CMOS sensor. CCD sensors excel at ISOs up to 200, but in lower light conditions, exposure time must be increased and Forward Motion Compensation (FMC) has to be employed to avoid smearing the images. The CMOS sensor has an ISO range of up to 6400, which enables short exposures instead of using FMC.
\end{abstract}

This paper aims to evaluate the strengths of each of the sensor types based on real missions over a test field in Speyer, Germany, used for airborne camera calibration. The test field area has about 30 Ground Control Points (GCPs), which enable a perfect scenario for a proper geometric evaluation of the cameras. The test field includes both a Siemen star and scale bars to show any blurring caused by forward motion.

The result of the comparison showed that both cameras offer high accuracy photogrammetric results with post processing, including triangulation, calibration, orthophoto and DEM generation. The forward motion effect can be compensated by a fast shutter speed and a higher ISO range of the CMOS-based camera. The results showed no significant differences between cameras.

\section{INTRODUCTION}

Forward Motion Compensation was a necessary component for analog cameras, due to the fact that film grain is not a standard grid and the grain's tiny size makes the smallest aircraft movement directly visible in the end product, causing a smear. The lower ISO and the slower large format shutters make non digital photography at speeds similar to today's cameras impossible. The cameras needed sophisticated engineering to design and build pressure plates that used suction to hold the film material to the plate during exposure. At the same time, the pressure plates move against the aircraft's direction to compensate for the forward motion. FMC was a standard part of each metric airborne, large format camera that was in use at the time.

When the first digital cameras were introduced to the aerial market, FMC was considered a necessary component and engineers worked on ways to implement this technology into digital cameras as well. Moving a sensor during exposure was difficult, since large format cameras at that time were based on an array of several (four to nine) single medium format or smaller sensors and the images were stitched in production. One way to solve this was based on the sensor's CCD read out technology which enables the cameras to move information during the image capture (sensor integration) from one line to the next. This technology was quickly adopted and is known today as time delayed integration (TDI). TDI uses the physical principles of the sensor's readout, in which the data captured in each line of pixels is moved to the next line after the end of the exposure time. When using TDI, a single line starting from the top of the sensor, receives the data and stops the integration before the end of the exposure time. It then moves the data onto the next line. This is happening simultaneously in all lines during the whole exposure time. This principle can be used for a limited number of lines and produces the same results as physically moving the sensor.

When large format digital cameras replaced analog cameras, they usually offered TDI as a method of dealing with forward motion, while medium format cameras typically used fast shutter speeds (up to a 1/1600). For most projects where medium format cameras were used, (mostly in combination with LIDAR systems), the compensation based on shutter speed worked quite well and offered the same image quality as cameras based on FMC. In some cases, the image quality was even better than images captured with large format digital cameras that used TDI. Over time, due to market requests, some medium format aerial cameras manufacturers started to offer TDI or physical based FMC. Today, nearly all aerial metric cameras have an FMC function if their sensor is based on CCD technology. Despite the availability of FMC, there are a large number of aerial metric cameras users, who still compensate for forward motion by using shutter speed, as they prefer the more photogrammetric way of taking images and try to avoid any physical or electronic movement of the information during exposure.

Theoretically, a physical movement of the sensor could offer a higher performance to FMC as it is a linear function. The sensor movement has to be accelerated to the compensation speed of the forward motion and then it can work linearly with subpixel accuracy. TDI is not a physical linear function and it compensates with pixel accuracy in the case of $\mathrm{B} \& \mathrm{~W}$ and achromatic sensors. For color (RGB) sensors, which use a 
Bayer filter pattern, the information is shifted two lines at a time to maintain color filter registration.

In 2014, a new sensor technology entered the market and offered new possibilities for medium format aerial cameras. These new CMOS sensors have a different pixel design and their readout is slightly different than the CCD sensors. Because of the way cameras read the data from CMOS sensors, TDI is not an option during exposure. Same as CCD sensors CMOS sensors use EM energy to generate pixel charges. But these charges are not transferred during the read out from pixel to pixel. In CMOS sensors all sensors in a column share a column bus and a sequential read out is done in a row serial order by an operation of switches. These are typical MOS field-effect transistors. This way to read out the pixels and not moving charges along a line don't allow a TDI based FMC. (Gerald Lepage)

Since CMOS sensors cannot be used for FMC, their inherent advantages sparked a new discussion about the need of FMC in airborne photogrammetric cameras and other real alternatives. CMOS sensors produce superb image quality when shooting at high ISOs, which enable operators to use high shutter speeds, (up to a 1/1600 using leaf shutters), thus freezing the aircraft's movement during a capture. Another benefit of CMOS sensors is that they have a much faster read out speed, which means a large increase in the continuous capture rate, of up to one to two frames per second. This opens up the possibility of much higher overlapping, 80 to $90 \%$, which gives users the option to generate 3D colored point clouds directly out of images. Keeping these possibilities in mind, there might be no need for FMC based on TDI, if compensation using fast shutter speeds could be an alternative. This new CMOS technology allows for a much larger portfolio of data generated from aerial images.

To directly compare a CMOS-based camera against a CCDbased camera offering TDI , Phase One Industrial and GGS $\mathrm{GmbH}$ performed a test flight over a camera calibration field in Speyer, Germany,. The flight parameters where set to the maximum to bring the CMOS based camera to the edge of compensation using shutter speed and high ISO to simulate real project conditions.

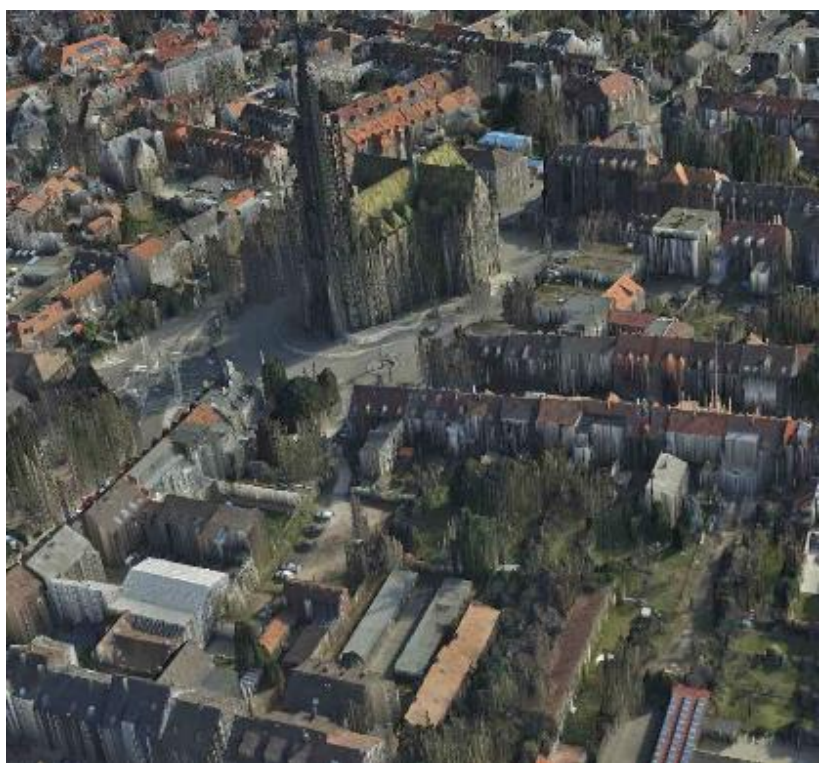

Figure 1. Colorized DSM of test area

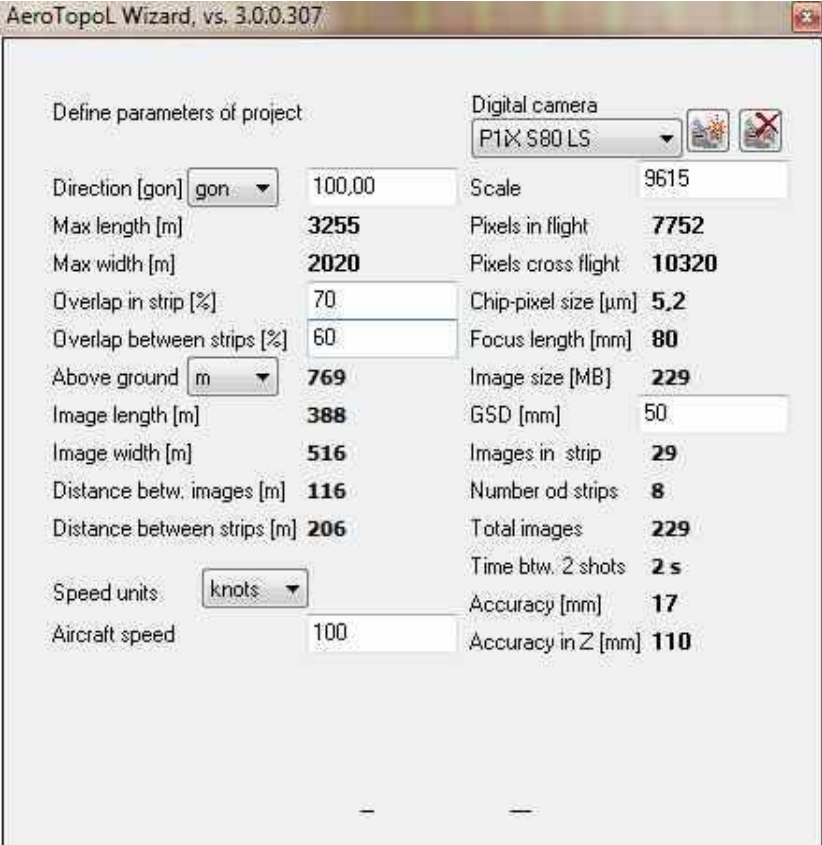

Figure 2. 5 CM GSD flight planning

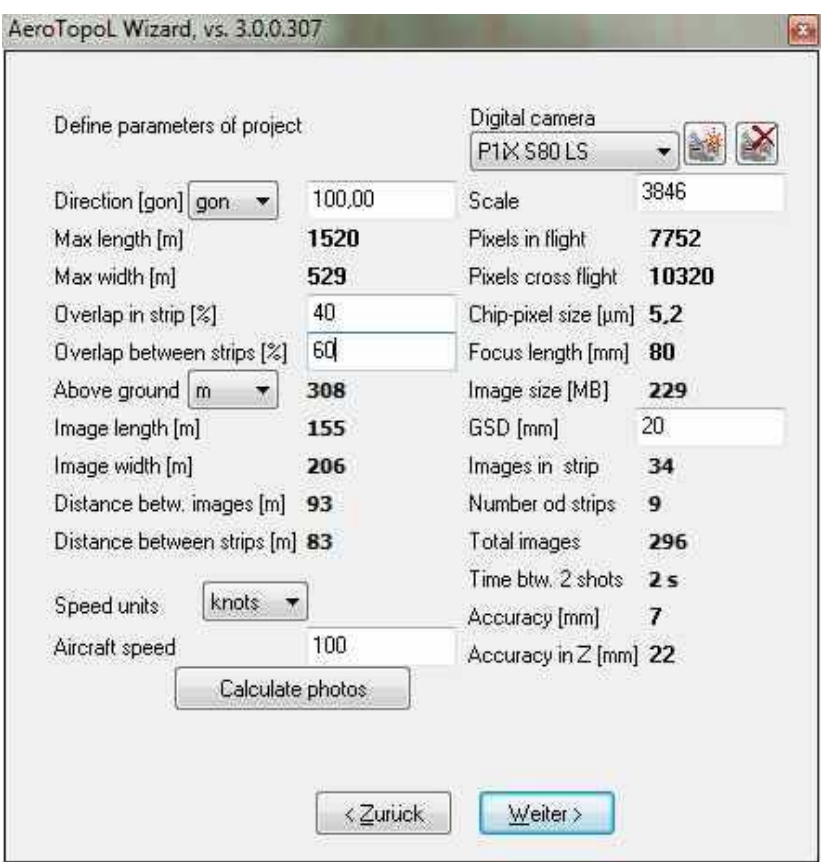

Figure 3. 2 CM GSD flight planning

\section{PROJECT PARAMETERS AND FLIGHT TEST}

GGS GmbH constructed a calibration field for airborne cameras with approximately 240 GCPs, which are measured with the latest GNSS technology to the highest accuracy. This GCP test field is spread out over the entire city and in addition, offers a variety of elevation points. The test field size is around 3,255 by 2,020 meters with an average terrain height of 110 meters above sea level. The coordinate system used for the field is based on GK 3, Bessel (Germany). GGS also placed black and white targets with different scales on top of their office building. The targets were placed in two directions, one along the flight lines and one across. In addition, a Siemens star was employed to evaluate the resolution of both cameras used in the project. The different scaling and directions of the targets 
enabled a clear visual inspection of the resolution and the FMC effect for both cameras.

Two cameras were used in the project: a Phase One iXA 180 with an 80 MP CCD sensor equipped with the FMC option and having an $80 \mathrm{~mm}$ Schneider-Kreuznach lens. The second cameras was a Phase One iXU-R 1000 with a 100 MP CMOS sensor, without an FMC option and having a $70 \mathrm{~mm}$ Rodenstock lens. Because of the different pixel sizes of each sensor, each camera offers nearly the same ground sample distance (GSD) for each camera at the same altitude. Both cameras were triggered simultaneously, but each camera used its own mid-exposure pulse (MEP) for the captures.

\begin{tabular}{|l|c|c|c|}
\hline Camera & Resolution & Lens & Pixel size \\
\hline iXA 180 & $\begin{array}{c}10,328 \text { by } 7,760 \\
\text { pixel }\end{array}$ & $80 \mathrm{~mm}$ & $\begin{array}{c}5.2 \text { by } 5.2 \\
\mu \mathrm{m}\end{array}$ \\
\hline iXU-R 1000 & $\begin{array}{c}11,608 \text { by } 8,708 \\
\text { pixel }\end{array}$ & $70 \mathrm{~mm}$ & $\begin{array}{c}4.6 \text { by } 4.6 \\
\mu \mathrm{m}\end{array}$ \\
\hline
\end{tabular}

Table 1. Camera specs

The cameras were mounted side by side on an AeroStab-Twin gyro mount from GGS (G. Kemper), pointing nadir, with an offset between the cameras of approximately ten centimeters. AeroTopoL (Kemper et al.) flight management system (FMS) was used to calculate two missions, one with a GSD of five centimeters and one with a GSD of two centimeters, and to execute the planned mission during the project. To capture high accuracy exterior orientation, the AeroDiDos GNSS/IMU system, which is based on a Novatel solution using FSAS IMU, completed the whole set up.

Since the aircraft used for the test would not allow for a slower flight speed, the difference in the overlap could affect the analytical results. A decision was made not to use the two centimeter project during the analytical comparison.

The flight was executed on the March 1, 2016 around noon, under standard weather conditions for Germany at that time of the year.

\section{VISUAL RESULTS FOR FMC COMPARISON}

After the flights, a visual inspection of the images from the two cameras from both flights with five and two centimeter GSD was performed. The initial impression was that there were no difference in the image quality between both cameras for the five centimeter GSD test. The targets that appear in the images were printed out and a comparison showed no differences, while the bars on the targets showed clearly that a GSD of five centimeters was achieved. It appears that the GSD was even a bit better than the calculated GSD.

For the two centimeter GSD test, the results were identical to the five centimeter GSD test, with images of both cameras being visually similar.

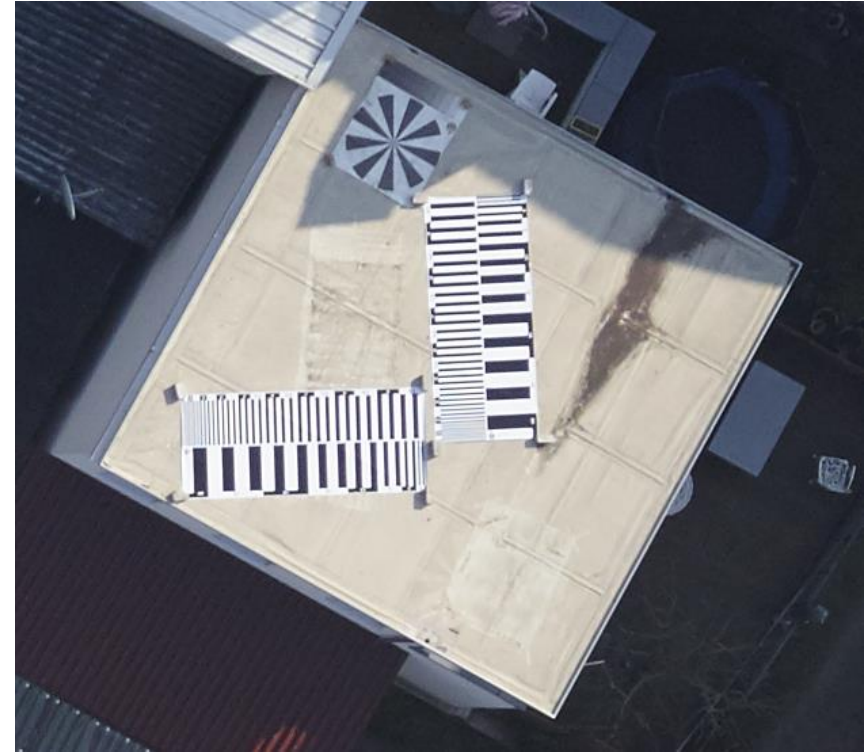

Figure 4. iXA 180 with FMC

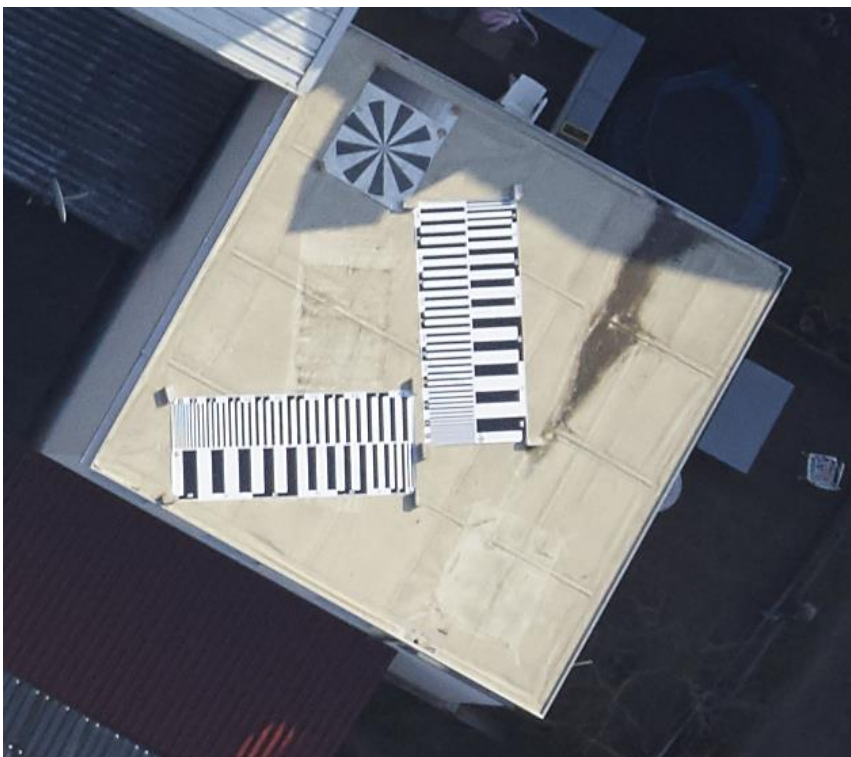

Figure 5. iXU-R 1000 without FMC

\begin{tabular}{|l|c|c|c|}
\hline Camera & ISO & $\begin{array}{c}\text { Shutter } \\
\text { speed }\end{array}$ & Aperture \\
\hline iXA 180 & 100 & $1 / 400 \mathrm{~s}$ & $\mathrm{~F} / 7.1$ \\
\hline iXU-R 1000 & 640 & $1 / 1600 \mathrm{~s}$ & $\mathrm{~F} / 7.1$ \\
\hline
\end{tabular}

Figure 6. Exposure parameters in detail

After an initial examination directly after the flight, it could be said that the non-FMC camera using a faster shutter speed and a higher ISO offers the same visual results and image quality as the camera using the FMC option based on TDI with a Bayer pattern sensor. 


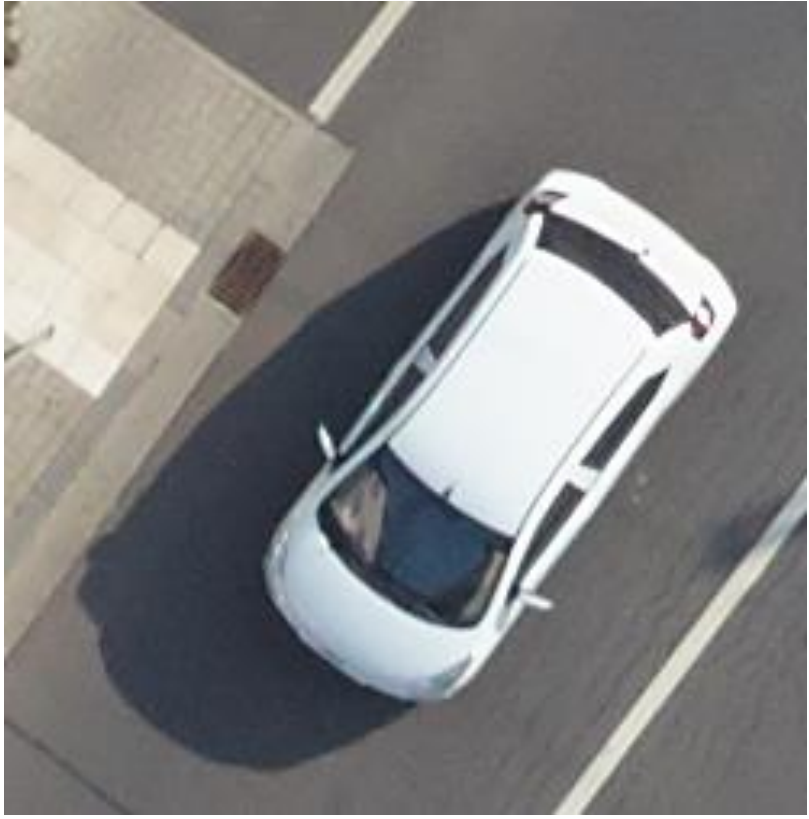

Figure 7. Detail of iXA 180 with FMC

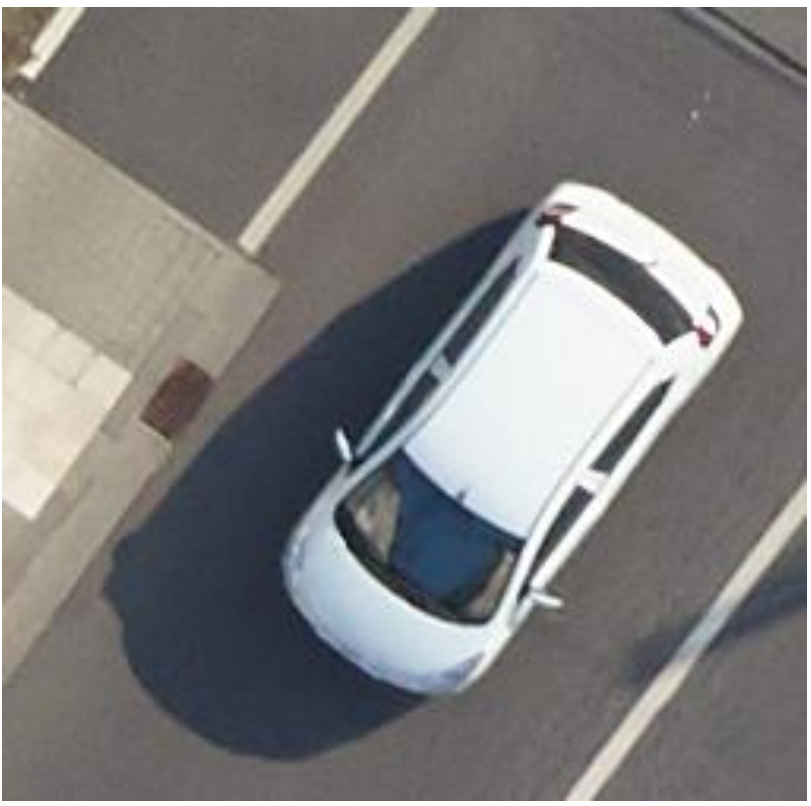

Figure 8. Detail of iXU-R 1000 without FMC

\section{ANALYTICAL RESULTS FROM POST PROCESSING}

The next step was to compare both camera types after a full post processing was performed of the two data sets. To do this, all acquired data was used: images, calibration data, GNSS/IMU data and 30 GCPs. The GCPs were measured with GNSS observations at a high accuracy with a standard deviation of $1.5 \mathrm{~cm}$.

Two post processing projects were performed, one with the iXA 180 and the other with the iXU-R 1000 and were based on a five centimeter GSD. Two additional post processing projects were performed, with the same cameras and were based on a two centimeter GSD. The results showed that the maximum continuous capture rate of the iXA 180 and the flight speed of the aircraft minimized the overlapping and affected the ability to compare results.
For the iXA 180 with the FMC option, 372 images were used the aerial triangulation. This resulted in 372 camera stations, an average flying altitude of 601.735 meter, a ground resolution of 0.038 meter/pixel, a coverage area of $6.848 \mathrm{sq} \mathrm{km,} \mathrm{3,271,968}$ tie-points, $9,476,127$ projections and an error of 0.6862 pixels.

The 30 ground control points were measured with: 0.016 meter in $\mathrm{X}, 0.024$ meter in $\mathrm{Y}$ and 0.192 meter in $\mathrm{Z}$ : with a total error of 0.432 in pixels for 220 observations.

Additional check points were used with the following overall results: 0.030 meter in $\mathrm{X}, 0.0366$ meter in $\mathrm{Y}$ and 0.074 meter in $\mathrm{Z}$ : with a total error of 0.661 pixels.

For the iXU-R 1000 without FMC function 515 images were used for the aerial triangulation. This resulted in 515 camera stations, an average flying altitude of 530.068 meter, a ground resolution of 0.034 meter/pixel, a coverage area of 7.2190 sq. $\mathrm{km}, 5,087,804$ tie-points, $14,728,935$ projections and an error of 0.7703 pixels.

The average camera location error was determined with: 0.396 meter in $\mathrm{X}, 0.188$ meter in $\mathrm{Y}$ and 1.483 meter in $\mathrm{Z}$ : with a total error of 1.546 meter.

The 30 Ground Control Points were measured with: 0.022 meter in $\mathrm{X}, 0.026$ meter in $\mathrm{Y}$ and 0.081 meter in $\mathrm{Z}$ : with a total error of 0.556 pixels for 220 observations.

Additional check points were used with the following overall results: 0.028 meter in $\mathrm{X}, 0.031$ meter in $\mathrm{Y}$ and 0.069 meter in $\mathrm{Z}$ : with a total error of 0.689 pixel.

\section{IN-FLIGHT CAMERA CALIBRATION}

Beside the analytical evaluation, a full in-flight camera calibration was performed using the GCPs and triangulation results. The calibration was based on the Australis Calibration System developed by Prof. Clive Fraser from the University of Melbourne. (Fraser et al) The cameras were calibrated by Phase One using the same calibration model. The difference between the Phase One lab calibration and the in-flight calibration was within calibration tolerance. (Kemper et al.) Cross lines were not flown, but images from a lower altitude were used from the flight with two centimeter GSD.

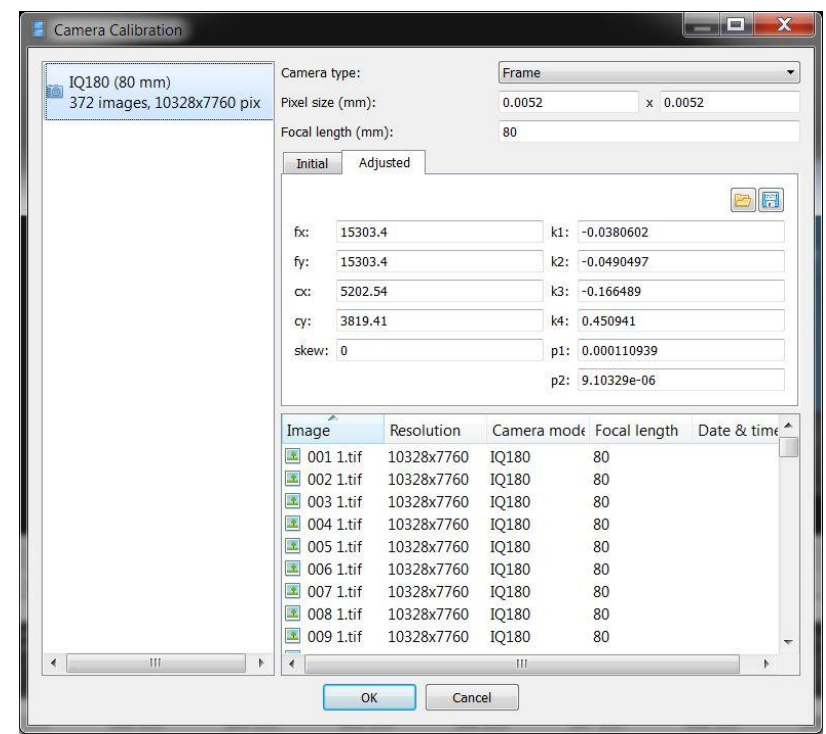

Figure 9. Calibration results for iXA 180 


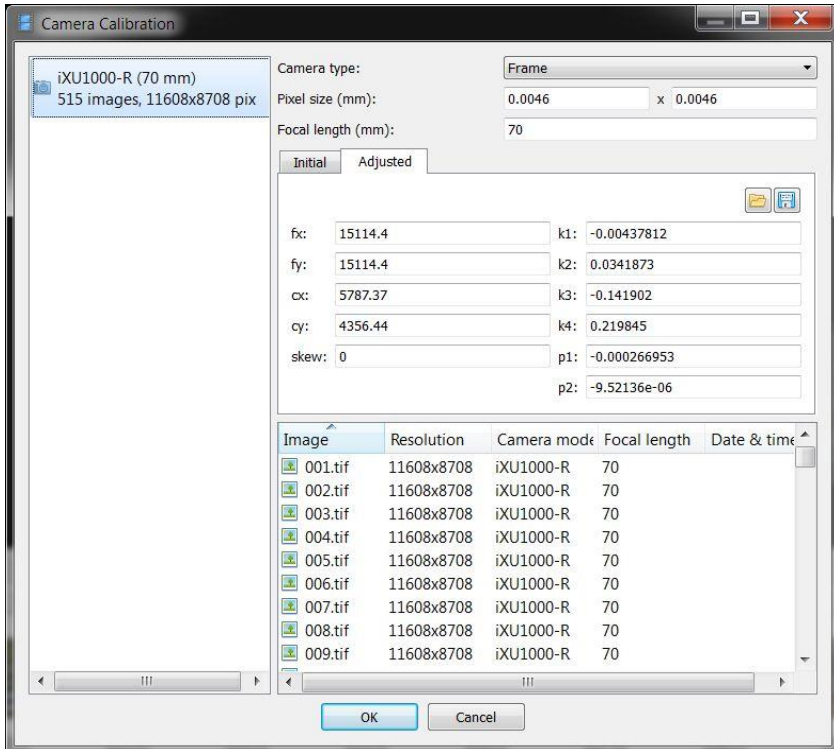

Figure 10. Calibration results for iXU-R 1000

\section{CONCLUSIONS}

The iXU-R 1000 offers a wide opening angle, a fast continuous capture rate and can easily compensate forward motion by working with a fast shutter speed and a higher ISO. In cases where a lower GSD is required, the alternative is to fly slower or to use a platform with FMC, such as the iXU 180 camera. Phase One aerial cameras that are equipped with the FMC option can easily compensate the flight speed by using the FMC.

When looking at the results from the different investigations, it appears that FMC has some benefits. However, based on the results, FMC is not necessary for most of the projects executed today in aerial photogrammetry. Both cameras, either with FMC using CCD sensor technology or without FMC using CMOS sensor technology, offer good results and neither of them has a real advantage over the other. The results from both cameras show their strengths and either camera may excel over the other in certain projects, where flight conditions may require the relative qualities of one over the other.

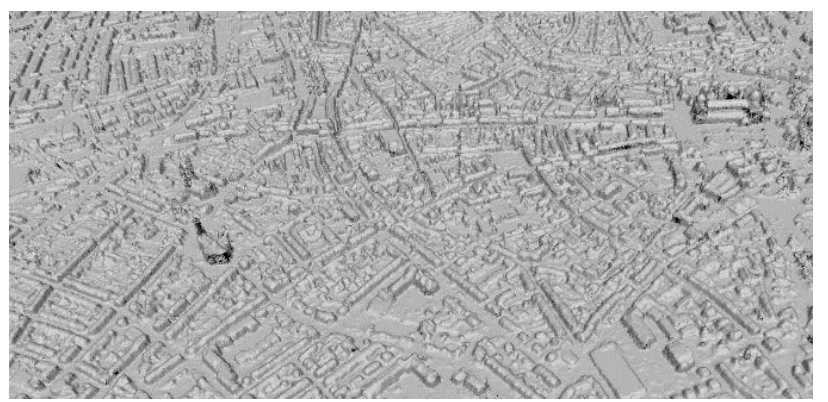

Figure 11. DSM of test area

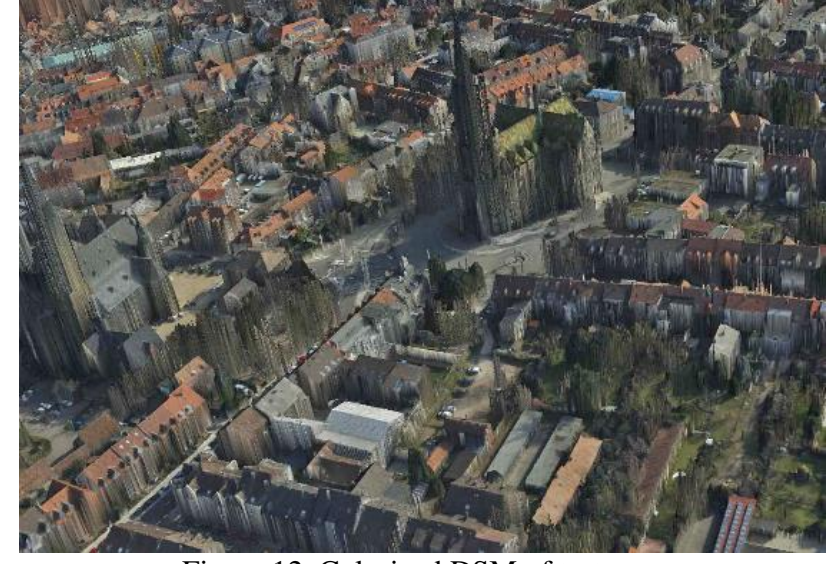

Figure 12. Colorized DSM of test area

It is also important to compensate for the roll of the aircraft when shooting with lower shutter speed, as the roll can affect the image quality the same way as forward motion does. A well balanced and properly working gyro mount is needed as part of the complete set up. Having this set up in place, the Phase One camera equipped with FMC can be used for low GSD imaging of one or two centimeters for any kind of project, even when using standard aircraft flying at typical cruising speeds. The camera's maximum continuous capture rate should be taken into consideration as well as the necessary overlaps. However, most of these projects are executed in combination with LIDAR systems and in these cases, a lower overlapping rate is needed.

Another consideration is the different lenses that were used for the test. Each of the two cameras used, had different lenses to capture the same GSD from the same altitude during the flight under the same environmental conditions. The different lens distortions can be taken out of the calibration data. Generally speaking, it could be said that the lens resolution is nearly identical for the $70 \mathrm{~mm}$ Rodenstock and the $80 \mathrm{~mm}$ SchneiderKreuznach lenses.

The analytical tests for both cameras showed a good photogrammetric result for the project. Both cameras finished the project with similar results. The elevation accuracy showed a lower result for both cameras, which was expected because of the relatively long focal lengths used. To increase the elevation accuracy, a different camera set up using shorter focal length could improve the results. The Phase One cameras offer exchangeable lenses, with a wide opportunity to adjust the H/B ratio to the required accuracy. Lenses like the Phase One Rodenstock $40 \mathrm{~mm}$ could easily turn this around.

As a final conclusion, it can be said that both cameras delivered the expected results regarding image quality, accuracy and performance. When looking at the analytical results from the five centimeter project, the expected $1 / 3$ to $1 / 2$ pixel resolution could be achieved as an overall accuracy. This proves that the cameras are photogrammetric survey products and with adding one or two crosslines, an even higher accuracy could be possible. However, for most standard projects in aerial photogrammetry, this would not even be required.

\section{REFERENCES}

Gerald Lepage (2008) Time Delayed Integration CMOS Image Sensor with Zero Desynchronization.

Fraser C, Stamatopoulos C. Automated target-free camera calibration. ASPRS 2014 Annual Conference: Geospatial 
Power in Our Pockets, Co-Located with Joint Agency Commercial Imagery Evaluation Workshop, JACIE 2014. 2014.

KEMPER, G., PIVNICKA, F., (2003): AeroTopoL - A system for planning, navigation and managing of aerial photo campaigns or scanning operations. 9th Conference about Information system for Agriculture and Forestry, proceedings, Prague / CZ.

Kemper, G., (2006): New airborne Sensors and Platforms for solving experimental Applications in Photogrammetry and Remote Sensing. Fifth International Symposium "TurkishGerman Joint Geodetic Days”, Berlin.

Kemper, G., Li Hongbo, Pauly, K. † (2008): New airborne Sensors and Platforms for specific applications in Photogrammetry and remote sensing; Proceedings of the ISPRS Congress 2008 in Beijing.

Hine, D., Kemper, G., Pivnicka, F., Li Hongbo (2008): Innovation in Flight Management Systems using real-time topological GIS Analysis; Proceedings of the ISPRS Congress 2008 in Beijing.

Kemper, G. (2010): Neue luftgestützte Sensoren und Plattformen für verschiedenste Aufgaben in der Fernerkundung. 3 Ländertagung der DGPF, OVG und SGPBF, Wien.

Kemper, G., Pivnicka, F., Geissler, S. (2012): Calibration Procedures in Mid Format Camera Setups; XXII ISPRS Congress, ISPRS Proceedings, Melbourne/ Australia. 